\title{
BMJ Open Potentially inappropriate prescribing and its association with health outcomes in middle-aged people: a prospective cohort study in Ireland
}

\author{
Frank Moriarty, ${ }^{1}$ Caitriona Cahir, ${ }^{2}$ Kathleen Bennett, ${ }^{2}$ Carmel M Hughes, ${ }^{1,3}$ \\ Rose Anne Kenny, ${ }^{4}$ Tom Fahey ${ }^{1}$
}

To cite: Moriarty F, Cahir C, Bennett K, et al. Potentially inappropriate prescribing and its association with health outcomes in middle-aged people: a prospective cohort study in Ireland. BMJ Open 2017;7:e016562. doi:10.1136/ bmjopen-2017-016562

- Prepublication history and additional material for this paper are available online. To view these files, please visit the journal online (http://dx.doi. org/10.1136/bmjopen-2017016562).

Received 24 February 2017 Revised 15 June 2017 Accepted 31 July 2017

CrossMark

${ }^{1}$ HRB Centre for Primary Care Research, Department of General Practice, Royal College of Surgeons in Ireland, Dublin, Ireland

${ }^{2}$ Division of Population Health Sciences, Royal College of Surgeons in Ireland, Dublin, Ireland

${ }^{3}$ Clinical and Practice Research Group, School of Pharmacy, Queen's University Belfast, Belfast, Northern Ireland

${ }^{4}$ The Irish Longitundinal Study on Ageing, Trinity College Dublin, Dublin, Ireland

Correspondence to

Dr Frank Moriarty;

frankmoriarty@rcsi.ie

\section{ABSTRACT}

Objectives To determine the prevalence of potentially inappropriate prescribing (PIP) in a cohort of communitydwelling middle-aged people and assess the relationship between PIP and emergency department (ED) visits, general practitioner (GP) visits and quality of life (QoL). Design Prospective cohort study.

Setting The Irish Longitudinal Study on Ageing (TILDA), a nationally representative cohort study of ageing. Participants Individuals aged $45-64$ years recruited to TILDA who were eligible for the means-tested General Medical Services scheme and followed up after 2 years. Exposure PIP was determined in the 12 months preceding baseline and follow-up TILDA data collection by applying the PRescribing Optimally in Middle-aged People's Treatments (PROMPT) criteria to participants' medication dispensing data.

Outcome measures At follow-up, the reported rates of ED and GP visits over 12 months (primary outcome) and the CASP-R12 (Control Autonomy Self-realisation Pleasure) measure of QoL (secondary outcome).

Analysis Multivariate negative binomial (rates) and linear regression (CASP-R12) models controlling for potential confounders.

Results At 2-year follow-up ( $\mathrm{n}=808$ ), PIP was detected in $42.9 \%$ by the PROMPT criteria. An ED visit was reported by $18.7 \%$ and $94.4 \%$ visited a GP (median 4 visits, IQR 2-6). Exposure to $\geq 2 \mathrm{PROMPT}$ criteria was associated with higher rates of healthcare utilisation and lower QoL in unadjusted regression. However, in multivariate analysis, the associations between PIP and rates of ED visits (adjusted incidence rate ratio (IRR) $0.92,95 \% \mathrm{Cl} 0.53$ to 1.58 ), and GP visits (IRR $1.06,95 \% \mathrm{Cl} 0.87$ to 1.28 ), and CASP-R12 score (adjusted $\beta$ coefficient $0.35,95 \% \mathrm{Cl}-0.93$ to 1.64 ) were not statistically significant. Numbers of medicines and comorbidities were associated with higher healthcare utilisation.

Conclusions Although PIP was prevalent in this study population, there was no evidence of a relationship with ED and GP visits and QoL. Further research should evaluate whether the PROMPT criteria are related to these and other adverse outcomes in the general middle-aged population.

\section{INTRODUCTION}

Potentially inappropriate prescribing (PIP) includes the use of medicines such that the
Strengths and limitations of this study

- This is the first study to assess the relationship between the PRescribing Optimally in Middle-aged People's Treatments criteria and health outcomes in a middle-aged population.

- Detailed information on dispensed medicines from an administrative pharmacy claims database was analysed.

- Participants in this study are not representative of the general middle-aged population due to their lower socioeconomic status.

- Exposure to potentially inappropriate prescribing (PIP) was measured over a fixed 12-month period prior to outcome measurement, so only sustained adverse effects of PIP would have been detected.

risks outweigh the benefits or where a safer alternative exists. ${ }^{12}$ Most research on PIP has focused on older patients (aged $\geq 65$ years), as they tend to be at greater risk of adverse drug events owing to changes in drug pharmacokinetics and pharmacodynamics that occur with ageing and increased likelihood of being on multiple medicines or having multiple conditions. ${ }^{1}$ Although chronological age is used as a marker for these factors, multimorbidity and polypharmacy are also prevalent in individuals in middle age (usually defined as between 45 and 64 years), particularly among socioeconomically deprived groups. ${ }^{34}$ Age-related physiological changes also do not occur at a single time point; rather, they develop on a continuum, with renal blood flow declining by $1 \%$ per year from the age of 50 years, for example. ${ }^{56}$ To date, there has been a paucity of research on PIP in middle-aged adults despite adverse drug events being more strongly associated with the numbers of medicines and diagnoses a person has than chronological age..$^{78}$

The PROMPT (PRescribing Optimally in Middle-aged People's Treatments) criteria 
have recently been developed as explicit indicators of PIP in middle-aged adults, specifying circumstances in which prescribing may be inappropriate. ${ }^{9}$ The risk of adverse effects from prescribing considered to be potentially inappropriate is often assumed to be lower in younger people than in individuals over 65 years, ${ }^{8}$ because superior baseline levels of physiological functioning generally mean higher drug concentrations are required in younger people to reach an adverse event-threshold effect. ${ }^{10}$ However, individual patient characteristics, such as comorbidities and the number of medicines being taken, may be more important than actual age in affecting drug response. ${ }^{6}$ Risk of adverse events for medicines included in PIP criteria can be similar in older and middle-aged groups after controlling for patient characteristics, as has been found, for example, with non-steroidal anti-inflammatory drugs (NSAIDs). ${ }^{11}$

The PROMPT criteria are a relatively new measure of PIP and although the prevalence has been evaluated, any relationship with important outcomes for patients has yet to be investigated..$^{212}$ It is necessary to determine if exposure to the PROMPT criteria has an impact on middleaged patients' outcomes, independent of any effect of multimorbidity and polypharmacy, to justify the use of these criteria as a process measure of prescribing quality or as part of any intervention to reduce PIP.

The aims of this study were: (1) to determine the prevalence of PIP using the PROMPT criteria in a cohort of community-dwelling middle-aged people, (2) to determine factors associated with change in prevalence over time in this cohort, and (3) to assess the prospective relationship between PIP and the outcomes of healthcare utilisation and quality of life (QoL).

\section{METHODS}

\section{Study design and setting}

This prospective cohort study included participants from The Irish Longitudinal Study on Ageing (TILDA), which is a nationally representative cohort study examining the health, economic and social circumstances of middleaged and older people living in the community. ${ }^{13}$ TILDA recruited approximately 8000 people in Ireland, based on a random selection process using a national geodirectory of residential addresses. While the target population was community-dwellers aged 50 years or over, participants' spouses and partners of any age were also invited to take part. Participants were asked to consent to use of their administrative pharmacy claims data from the Health Service Executive Primary Care Reimbursement Service (HSE-PCRS). Participants were included in the present study if they (1) were aged 45 years or older at baseline (Wave 1) TILDA interview and 64 years or less at follow-up (Wave 2) interview, (2) were eligible for the General Medical Services (GMS) scheme, and (3) provided a GMS identifier which was successfully linked to their pharmacy claims data (see figure 1). The GMS scheme is public health cover in Ireland providing free health services, including most prescribed medicines, to those who are eligible, although a small monthly copayment per prescription item was introduced in 2010. Eligibility for the GMS scheme is based on means testing (covering approximately $33 \%$ of the $45-64$ years age group in 2012) and so this cohort is considered more socioeconomically deprived than the general middle-aged population. ${ }^{14}$ The STROBE (Strengthening the Reporting of Observational Studies in Epidemiology) standardised reporting guidelines for cohort studies have been followed in the reporting of this research. ${ }^{15}$

\section{Data collection}

TILDA data collection is conducted in waves every 2 years incorporating a face-to-face interview, self-completion questionnaire and a health assessment. ${ }^{13}$ Baseline data collection was carried out from October 2009 to February 2011 and participants were followed up after approximately 2years between February 2012 and March 2013. Trained interviewers visited participants in their own homes where they conducted a computer-assisted personal interview (CAPI), which ensured consistent ordering and wording of questions, and full CAPI documentation is available on the TILDA website. Details of prescribed medicines that were dispensed were extracted from the HSE-PCRS pharmacy claims database for each participant in the present study from 15 months before the date of their TILDA baseline interview up to their follow-up interview and all identifiable information was removed after extraction. Ethical approval for TILDA was provided by the Faculty of Health Sciences Ethics Committee, Trinity College Dublin, and included provision for the secondary analysis of collected data and provision for linkage to participant's GMS dispensing information.

\section{Prevalence of PIP}

Application of PROMPT criteria

The prevalence of PIP using the PROMPT criteria was determined in this cohort during two time intervals: (1) the 12 months preceding the date of each participant's baseline TILDA interview and (2) the 12 months preceding their follow-up interview. PIP was assessed by applying the PROMPT criteria to participants' medicine dispensing data and information from TILDA on diagnoses and other characteristics. All 22 PROMPT criteria were applicable to the data available and each was assessed based on the same assumptions as in the previous paper applying the PROMPT criteria ${ }^{14}$; details of these and how various types of criteria were applied to the data are both provided in online supplementary box S1. Data were available for all participants to assess PIP prevalence during both time intervals. For criteria dependent on duration of use greater than 1 month, a longer period of time was analysed by including a lead-in period to allow for 12 months of potential exposure.

\section{Statistical analysis}

The prevalence of PIP for each time period was calculated as the proportion of the cohort with any PROMPT criterion, and this was also assessed by the number of PROMPT 
Participants recruited to TILDA at Wave 1 (baseline) $(n=8,504)$

\begin{tabular}{|c|c|}
\hline & $\begin{array}{l}\text { Participants aged less than } 45 \text { years at Wave } 1 \\
\text { (baseline) }(n=70)\end{array}$ \\
\hline$\downarrow$ & $\begin{array}{l}\text { Participants aged } 65 \text { years or over at Wave } 2 \\
\text { (follow up) }(n=4,603)\end{array}$ \\
\hline
\end{tabular}

Participants aged 45 years or over at Wave 1 (baseline) and less than 65 years at Wave 2 (follow up) $(n=3,831$ )

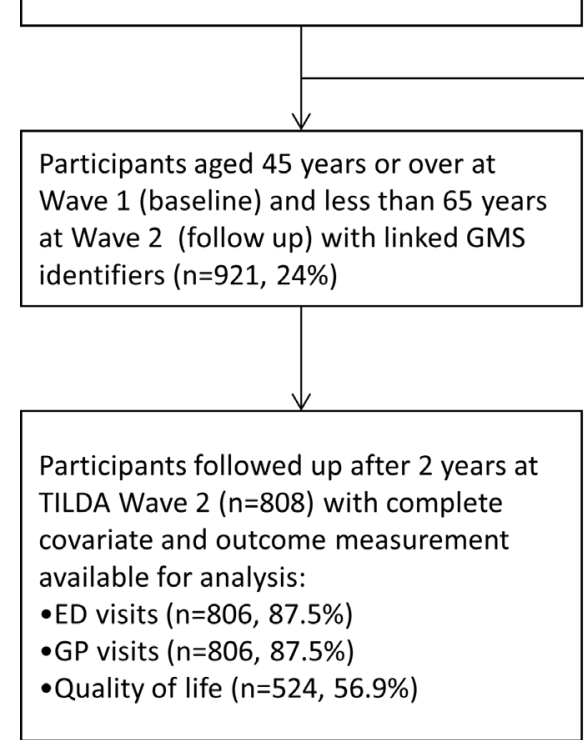

Figure 1 Flow diagram of study participants from The Irish Longitudinal Study on Ageing (TILDA) cohort aged 45-64 years. ED, emergency department; GP, general practitioner; GMS, General Medical Services.

criteria per individual (considering any number of duplicated drug classes as one PIP criterion). The proportion of the study cohort with each individual criterion was also determined. The absolute change in prevalence between these two periods (with 95\% confidence intervals (CI)) was calculated and McNemar's test for paired groups was used to assess if the overall prevalence and prevalence per criterion had changed significantly over time.

Generalised estimating equations (GEE) with exchangeable correlations were used to investigate the determinants of population-averaged change in PIP prevalence between the time periods. ${ }^{16}$ First, unadjusted GEE analysis was used to assess the odds of having any PIP at follow-up compared with baseline, and then multivariate analysis was performed which adjusted for factors shown previously to be associated with PIP (see Table S1), including age, sex and number of regular medicines, ${ }^{14}$ as well as reported number of doctor-diagnosed chronic conditions, ${ }^{17}$ and level of educational attainment as an indicator of socioeconomic status (SES) ${ }^{18}{ }^{19}$ Unadjusted and adjusted odds ratios (OR) with 95\% CI are presented.

\section{Association with patient outcomes}

\section{Outcomes}

The primary outcome was healthcare utilisation, specifically rates of emergency department (ED) visits and general practitioner (GP) visits. This was based on self-report and TILDA participants were asked during interview to recall how many times in the last 12 months they visited a hospital ED as a patient, and about how often they visited their GP.

The secondary outcome was QoL, which was assessed in the self-completion questionnaire which TILDA participants complete and return postinterview. The CASP-19 (Control Autonomy Self-realisation Pleasure) was the included measure, which was developed for use in studies of ageing and is applicable to older individuals and people in middle age. ${ }^{20}$ Participants rate how often each statement in the measure describes how they feel, giving a possible range from 0 (worst quality of life) to 57 (best quality of life). A revised 12-item version of CASP (CASPR12), which has been demonstrated to have improved psychometric validity, was used as the secondary outcome measure in this analysis. ${ }^{21}$

\section{Covariates}

The exposure of interest was PIP, specifically the number of PROMPT criteria that a participant was exposed to in the 12 months preceding outcome measurement. Participant demographics such as age, sex and level of educational attainment were included as potential confounders. As patients with polypharmacy and/or multimorbidity are 
more likely to be exposed to PIP, number of regular medicines and number of doctor-diagnosed chronic conditions were also included. ${ }^{1722}$ Covariates specific to each outcome were also considered, such as the number of ED/GP visits reported by the participants in 12 months preceding baseline interview for healthcare utilisation. For QoL, baseline CASP-R12 score and level of depressive symptoms screened using the Centre for Epidemiological Studies Depression scale (CES-D) at follow-up were considered. Further description of each covariate is included in online supplementary table S1.

\section{Statistical analysis}

The relationship between PIP and healthcare utilisation was assessed by fitting separate negative binomial regression models for the reported numbers of (1) ED visits in the 12 months preceding follow-up interview and (2) GP visits in the same period. PIP exposure was included as a variable for number of criteria, coded as 0 (reference), 1 and $\geq 2$ PROMPT criteria to investigate a potential doseresponse relationship. Univariate analysis was conducted, followed by multivariate analysis adjusting for potential confounders. For covariates that could vary over time, the value at the latest time point prior to outcome measurement was used. Results of these analyses are presented as incidence rate ratios (IRR) with $95 \%$ CI. A similar analytical approach was used for the secondary outcome of QoL using participants' CASP-R12 score measured at follow-up (possible range 0-36) as the continuous outcome variable. Univariate and multivariate linear regression analyses were conducted and results are presented as $\beta$ regression coefficients with $95 \%$ CI. Multicollinearity in the models was assessed using variance inflation factors. Two-sided statistical significance was assumed at $\mathrm{p}<0.05$. Analyses were performed using Stata V.13 (Stata Corporation, College Station, Texas, USA).

\section{Sensitivity analysis}

The impact of time-dependent confounding (by number of regular medicines or chronic conditions for example) was investigated using marginal structural models (MSMs). ${ }^{23}$ Two types of analytical weight were calculated for each participant. The first type was stabilised inverse probability weights, the inverse of the probability of having their PIP exposure conditional on past PIP exposure and covariate history (both baseline and time-varying). The second type was censoring weights, the probability of remaining uncensored (ie, being followed up at Wave 2) given past PIP exposure and covariate history. Weighted regression analyses (ie, MSMs) were performed for each outcome using the product of these weights, including a binary PROMPT exposure variable and baseline covariates only.

\section{RESULTS \\ Participants}

At baseline, 921 TILDA participants aged 45-64 years were included and of these, $808(87.8 \%)$ completed follow-up interview after 2 years (figure 1). Those followed up were predominantly female $(60.2 \%)$, had a mean age of 58.0 years (SD 4.0), a median of four regular medicines (IQR 1-6) and two reported doctor-diagnosed chronic conditions (IQR 1-3) (table 1).

\section{Prevalence of PIP and change over time}

Overall, 361 participants had at least one PROMPT criteria in the 12 months prebaseline interview (39.2\%).

\begin{tabular}{|c|c|c|}
\hline Characteristic & Baseline ( $n=921)$ & Follow-up $(n=808)$ \\
\hline Age (year, mean (SD)) & $56.11(4.13)$ & $58.01(4.06)$ \\
\hline Female sex (n (\%)) & $560(60.8)$ & $486(60.2)$ \\
\hline Number of regular medicines (median (IQR)) & $3(0-5)$ & $4(1-6)$ \\
\hline \multicolumn{3}{|l|}{ Number of reported conditions ( $\mathrm{n}(\%))$} \\
\hline 0 & $183(19.9)$ & $99(12.3)$ \\
\hline 1 & $260(28.2)$ & $190(23.5)$ \\
\hline 2 & $240(26.1)$ & $198(24.5)$ \\
\hline $3+$ & $238(25.8)$ & $321(39.7)$ \\
\hline \multicolumn{3}{|l|}{ Level of education attainment $(\mathrm{n}(\%))$} \\
\hline Primary & $320(34.7)$ & $265(32.8)$ \\
\hline Secondary & $443(48.1)$ & $399(49.4)$ \\
\hline Tertiary & $158(17.2)$ & $144(17.8)$ \\
\hline \multicolumn{3}{|l|}{ Depressive symptoms ${ }^{*}(\mathrm{n}(\%))$} \\
\hline None & $517(57.1)$ & $492(61.7)$ \\
\hline Subclinical & $200(22.1)$ & $157(19.7)$ \\
\hline Clinical & $189(20.9)$ & $148(18.6)$ \\
\hline
\end{tabular}

*Missing for 15 participants (1.6\%) at baseline, and 11 (1.4\%) at follow-up. 
Considering number of PROMPT criteria, $21.6 \%$ of participants had one, $10.3 \%$ had two and $7.3 \%$ had three or more criteria in this time period (table 2). In the 12 months preceding follow-up interview, the prevalence had increased significantly to $42.9 \%$, with the breakdown by number of criteria for one, two and three or more being $22.1 \%, 11.9 \%$ and $8.9 \%$, respectively.

As shown in table 2, the most prevalent PIP criteria at baseline were long-term PPIs at higher than maintenance dose (prescribed to $14.8 \%$ of participants), strong opioids without a laxative (13.9\%), long-term use of benzodiazepines $(7.6 \%)$, of non-benzodiazepine or Z-drug hypnotics $(7.6 \%)$ and of NSAIDs $(6.2 \%)$. Of these, statistically significant increases in prevalence over the 2-year period were observed for long-term PPIs above maintenance dose (to $19.2 \%$, McNemar's test $\mathrm{p}<0.001$ ), long-term use of non-benzodiazepine hypnotics (to $9.2 \%, \mathrm{p}<0.05$ ) and of NSAIDs (to $8.3 \%, \mathrm{p}<0.05$ ), as well as for duplicate opioid prescribing (from $0.8 \%$ to $2.3 \%, \mathrm{p}<0.01$ ). The rank order of the most prevalent criteria did not change between the two time periods. Although most criteria increased in prevalence, a number did decrease, although not significantly, such as use of NSAIDs without gastroprotection with low-dose aspirin (from $4.3 \%$ to $2.2 \%$ ) or with selective serotonin reuptake inhibitors (from $2.8 \%$ to $1.6 \%$ ), and duplicate benzodiazepines (from $2.2 \%$ to $1.3 \%$ ) or NSAIDs (from $1.0 \%$ to $0.5 \%$ ).

Analysis of factors associated with change in prevalence as shown in table 3 found that while the overall prevalence increased significantly at follow-up (unadjusted OR $1.08,95 \%$ CI 1.01 to 1.15 , for follow-up compared with baseline), this was no longer significant after adjusting for patient factors (adjusted OR 0.93, 95\% CI 0.85 to 1.02). Numbers of medicines and diagnosed conditions, and female sex were all significantly associated with a higher odds of PIP in the multivariate analysis, and tertiary level education (relative to primary) was associated with lower odds of PIP (table 3 ).

\section{Association with patient outcomes}

At follow-up interview, $18.7 \%$ of the cohort reported visiting an $\mathrm{ED}$ in the previous 12 months, and of these, $13.1 \%$ of participants reported one ED visit, $2.9 \%$ reported two and $2.7 \%$ reported three or more. During the same time frame, $94.4 \%$ of participants reported visiting a GP (median four visits, IQR 2-6).

In univariate negative binomial regression, having two or more PROMPT criteria was associated with an increased rate of ED visits (unadjusted IRR 1.98, 95\% CI 1.27 to 3.08) relative to having no criteria but in the multivariate model adjusting for confounders (table 4), this association was no longer significant (adjusted IRR 0.92, 95\% CI 0.53 to 1.58 ). Increasing age and tertiary education (relative to primary) were significantly associated with a lower rate of ED visits, while number of regular medicines was associated with an increased ED visit rate.

Unadjusted analysis of GP visits showed an apparent dose-response relationship, with an increased rate associated with having one PROMPT criterion (unadjusted IRR $1.35,95 \%$ CI 1.17 to 1.56 ) or two or more criteria $(1.89,95 \%$ CI 1.62 to 2.21) (table 4). However, these associations were not significant after controlling for confounders in multivariate analysis. Number of regular medicines, having three or more reported conditions (relative to none) and number of GP visits at baseline were all significantly associated with a higher rate of GP visits at follow-up in multivariate analysis.

CASP-R12 scores at follow-up ranged from 6 to 36 and the mean score was 24.8 (SD 5.8). The distribution was approximately normal and in model checking, none of the assumptions of linear regression were violated. Exposure to PIP was associated with a reduction in CASP-R12 QoL score in univariate analysis compared with those with no PIP (table 4), regardless of whether participants had one PROMPT criterion (unadjusted $\beta$ coefficient -1.70 , $95 \% \mathrm{CI}-2.97$ to -0.44$)$ or two or more criteria $(-2.13$, $95 \% \mathrm{CI}-3.45$ to -0.80 ). This two unit reduction in CASPR12 score is equivalent to answering two positively worded statements 'Rarely' instead of 'Sometimes', for example. After controlling for confounders, there was no evidence of a significant reduction in QoL associated with any PIP exposure. Female sex, increasing age and higher baseline CASP-R12 score were significantly associated with higher follow-up CASP-R12 score, while presence of depressive symptoms (both subclinical and clinical) was associated with lower QoL.

In sensitivity analysis using MSMs (see online supplementary table S2), although changes occurred in the point estimates of adjusted IRR for ED visits (from 0.95 to 0.77 ) and adjusted $\beta$ coefficient for CASP-R12 score (from -0.26 to -0.07 ), associations remained non-significant and so adjusting for time-varying confounding did not significantly alter the findings.

\section{DISCUSSION}

\section{Principal findings}

The prevalence of PIP in these middle-aged TILDA participants was high at $39 \%$ in the 12 months preceding baseline interview and this had increased significantly to $43 \%$ at follow-up after 2 years. The increase in odds of having PIP at follow-up compared with baseline was not statistically significant once participant characteristics, such as number of medicines and chronic conditions, were accounted for. Although exposure to the PROMPT criteria was associated with outcomes in univariate analysis, no significant relationship between PIP and rates of ED and GP visits or CASP-R12 score was found once other factors were controlled for. The number of regular medicines was independently associated with higher rates of both ED and GP visits, while having three of more chronic conditions was associated with an increased rate of GP visits.

\section{Findings in the context of previous research}

PIP in middle-aged groups has been under-investigated; however, previous studies evaluating other forms of 
Table 2 Number and percentage of participants with each PRescribing Optimally in Middle-aged People's Treatments (PROMPT) criterion and change in percentage prevalence between baseline and 2-year follow-up

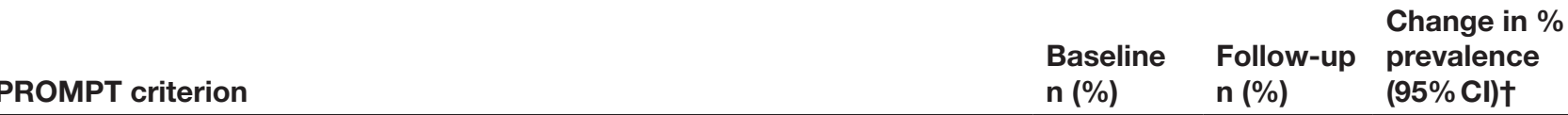

Gastrointestinal system

Other than for opioid-induced constipation, stimulant laxatives (eg, $\quad \begin{array}{lll}0(0.0) & 0(0.0) & 0.0(-0.1,0.1)\end{array}$

bisacodyl, senna) should not be prescribed as first-line treatment in

constipation for greater than 4 weeks

Proton pump inhibitors (PPIs) should not be prescribed at doses above the $136(14.8) \quad 177(19.2) \quad 4.5(2.1,6.8)^{\star \star \star} \neq$

recommended maintenance dosage for greater than 8 weeks

Esomeprazole or omeprazole should not be used in combination with $\quad 11(1.2) \quad 7(0.8) \quad-0.4(-1.2,0.3)$

clopidogrel

Cardiovascular system

The use of alpha-adrenoceptor blocking drugs (eg, doxazosin, prazosin) as $2(0.2) \quad 2(0.2) \quad 0.0(-0.4,0.4)$ monotherapy for hypertension should be avoided

$\begin{array}{llll}\text { Aspirin doses should not exceed } 150 \mathrm{mg} / \text { day for antiplatelet therapy } & 5(0.5) & 5(0.5) & 0.0(-0.4,0.4) \\ \text { Cardioselective calcium-channel blockers (eg, verapamil, diltiazem) should } & 4(0.4) & 4(0.4) & 0.0(-0.4,0.4)\end{array}$

Cardioselective calcium-channel blockers (eg, verapamil, diltiazem) should $\quad 4(0.4) \quad 4(0.4) \quad 0.0(-0.4,0.4)$ not be used in combination with beta-adrenoceptor blocking drugs
The use of oral short-acting dipyridamole should not be used as
$0(0.0) \quad 0(0.0)$
$0.0(-0.1,0.1)$

monotherapy in antiplatelet treatment

Respiratory system

First-generation antihistamines (eg, chlorphenamine, promethazine) should $8(0.9) \quad 5(0.5) \quad-0.3(-1.1,0.5)$

not be used as first-line agents for greater than 7 days.

A concomitant bisphosphonate should be prescribed if oral corticosteroids $14(1.5) \quad 14(1.5) \quad 0.0(-1.1,1.1)$ are used long term (greater than 3 months)

Theophylline should not be used as monotherapy for asthma or COPD $\quad 1(0.1) \quad 1(0.1) \quad 0.0(-0.4,0.4)$

Mucolytic agents (eg, carbocisteine, mecysteine) should not be used $\quad 1(0.1) \quad 6(0.7) \quad 0.5(0.0,1.1)$

routinely in stable COPD

Central nervous system

Selective serotonin reuptake inhibitors (SSRIs) should not be used in $\quad 1(0.1) \quad 0(0.0) \quad-0.1(-0.4,0.2)$

combination with venlafaxine

Tricyclic antidepressants (TCAs) should not be used as first-line in treatment $\quad 0(0.0) \quad 0(0.0) \quad 0.0(-0.1,0.1)$

of depression

Benzodiazepines should not be used long term (greater than 4 weeks) $\quad 70(7.6) \quad 76(8.3) \quad 0.6(-0.9,2.2) \ddagger$

Non-benzodiazepine hypnotics (zolpidem, zaleplon, zopiclone) should not be $70(7.6) \quad 85(9.2) \quad 1.6(0.0,3.3)^{*} \ddagger$ used long term (greater than 4 weeks)

Carbamazepine should not be used in combination with clarithromycin or $3(0.3) \quad 1(0.1) \quad-0.2(-0.8,0.3)$ erythromycin

Strong opioids (eg, buprenorphine, diamorphine, fentanyl, morphine, $\quad 128(13.9) \quad 131(14.2) \quad 0.3(-2.4,3.0) \neq$ oxycodone) should not be prescribed without the coprescribing of at least

osmotic or stimulant laxative (same month)

Infections
Nitrofurantoin should not be prescribed for greater than 7 days for the
$2(0.2)$
$8(0.9)$
$0.7(-0.1,1.4)$
management of uncomplicated lower urinary tract infections

Endocrine system

In relation to the management of diabetes, the use of oral long-acting

sulfonylureas (glibenclamide) should be avoided

$0(0.0) \quad 0(0.0) \quad 0.0(-0.1,0.1)$

Musculoskeletal system

\begin{tabular}{|c|c|c|c|}
\hline $\begin{array}{l}\text { Non-steroidal anti-inflammatory drugs (NSAIDs) should not be used long } \\
\text { term (greater than } 3 \text { months) }\end{array}$ & $57(6.2)$ & $76(8.3)$ & $2.1(0.3,3.8)^{*} \ddagger$ \\
\hline $\begin{array}{l}\text { Unless GI protection is provided with } \mathrm{PPI} / \mathrm{H}_{2} \text {-receptor antagonist, NSAIDs } \\
\text { should not be used in combination with low-dose aspirin }\end{array}$ & $40(4.3)$ & $20(2.2)$ & $-2.2(-2.2,0.7) \ddagger$ \\
\hline
\end{tabular}




\section{PROMPT criterion}

Unless $\mathrm{Gl}$ protection is provided with $\mathrm{PPI} / \mathrm{H}_{2}$-receptor antagonist, NSAIDs should not be used in combination with SSRIs

Duplication of drug classes

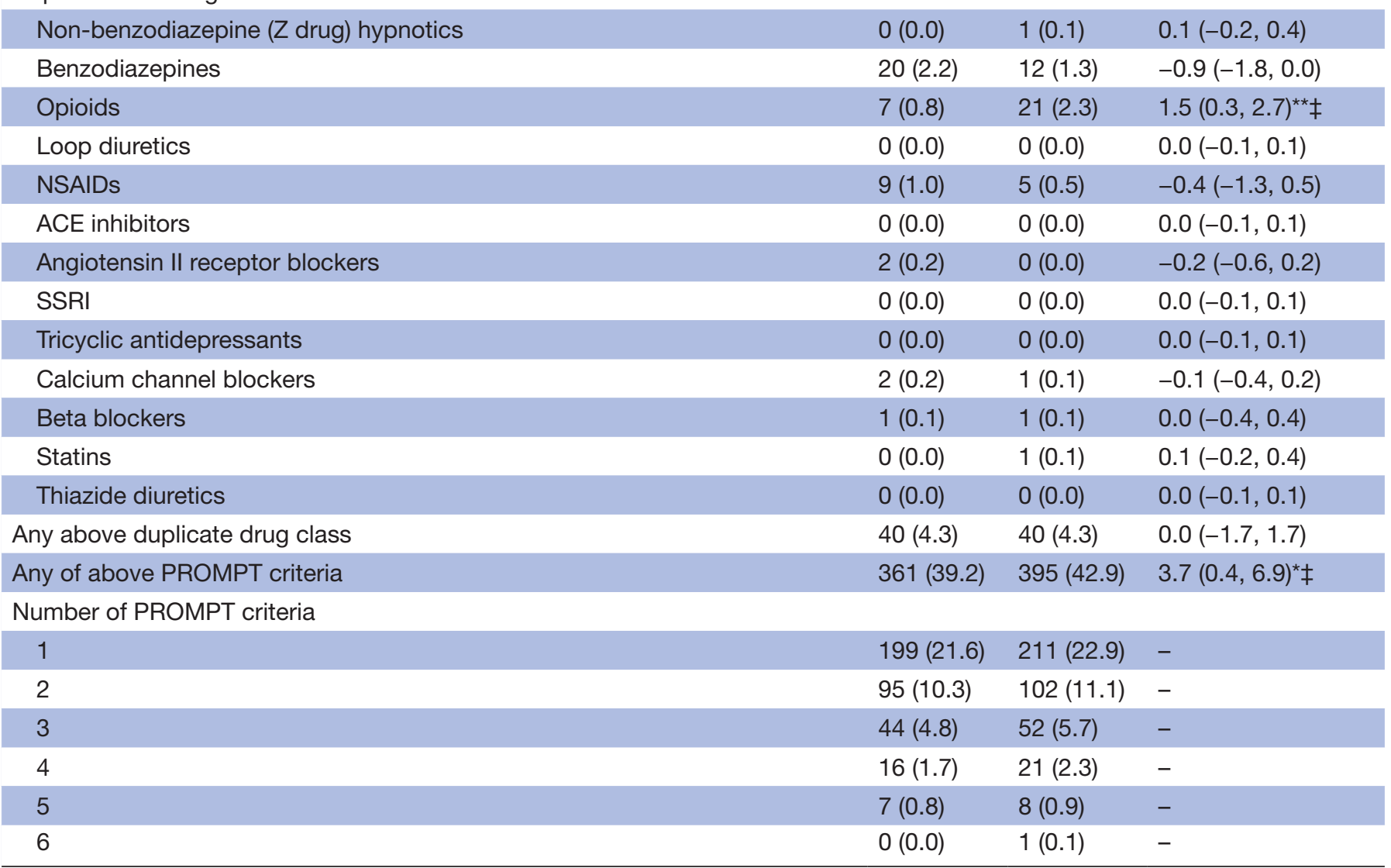

†Change in prevalence may not sum to exact difference in reported baseline and follow-up prevalence due to rounding.

${ }^{* * *} p$ value $<0.001 ;{ }^{* *} p$ value $<0.01 ;{ }^{*} p$ value $<0.05$.

$\ddagger$ Significant McNemar's test $(p<0.05)$ calculated using non-exact $p$ value if $>20$ individuals changed exposure status between time periods (ie, had criterion during one time period only).

ACE, angiotensin converting enzyme; COPD, chronic obstructive pulmonary disease; $\mathrm{H}_{2}$, histamine-2 receptor.

suboptimal prescribing have shown a similar risk of drugdrug interactions and high-risk prescribing across middle and older age groups after controlling for patient factors such as numbers of medicines and morbidity. ${ }^{425}$

No studies have evaluated the effect of PIP specifically on middle-aged people previously. There is evidence that PIP in older age is associated with adverse outcomes for patients, ${ }^{26}$ including from older participants of TILDA where similar analysis to the present study demonstrated a relationship between PIP criteria from the Screening Tool for Older Persons' Prescriptions and healthcare use among participants aged $\geq 65$ years. ${ }^{27}$ Prevalent PROMPT criteria here related to drug classes which are also commonly involved in PIP in older populations, including PPIs, NSAIDs and benzodiazepines. Some studies have reported a similar risk of adverse drug events resulting from these medicines in younger and older patients after adjustment for patient characteristics, such as gastrointestinal adverse events in trials of NSAIDs, ${ }^{11}$ and mortality risk among sedative hypnotic users. ${ }^{28}$

The relationship between age and harm from medicines has been described as ambiguous. ${ }^{6}$ Reporting age-specific rates of adverse drug events (ADEs) often indicates a plateau following childhood and a sharp rise in older age groups ${ }^{29}$; however, previous studies have indicated that this may be accounted for by increasing volumes of medicines consumed or patients' comorbidities. 83031 This supports the suggestion that an individual's physiological and clinical characteristics are more important than age in predicting adverse effects of drugs. ${ }^{6}$ However, evidence is equivocal, ${ }^{32}$ and differences between studies may be explained by classification of ADEs, for instance, there may be differences in seriousness of ADEs or preventability across age groups 3334 
Table 3 Factors associated with having any PRescribing Optimally in Middle-aged People's Treatments (PROMPT) criteria in population-averaged generalised estimating equations models

\begin{tabular}{|c|c|c|}
\hline & \multicolumn{2}{|c|}{ Prevalence of any PROMPT criteria $\left(n=919^{*}\right)$} \\
\hline & Unadjusted OR (95\% Cl) & Adjusted OR $(95 \% \mathrm{Cl})$ \\
\hline Follow-up (vs baseline) & $1.08(1.01$ to 1.15$) \dagger$ & 0.93 (0.85 to 1.02$)$ \\
\hline Age (years) & 1.05 (1.02 to 1.08$) \dagger$ & 1.02 (0.99 to 1.05$)$ \\
\hline Female (vs male) & $1.37(1.08$ to 1.74$) \dagger$ & $1.44(1.12$ to 1.85$) \dagger$ \\
\hline Number of regular medicinesł & $1.33(1.28$ to 1.40$) \dagger$ & $1.30(1.24$ to 1.37$) \dagger$ \\
\hline Number of chronic conditions $\ddagger$ & $1.46(1.35$ to 1.58$) \dagger$ & $1.12(1.01$ to 1.23$) \dagger$ \\
\hline \multicolumn{3}{|l|}{ Level of education (vs primary) } \\
\hline Secondary & 0.85 (0.66 to 1.09$)$ & 0.91 (0.69 to 1.19$)$ \\
\hline Tertiary & $0.68(0.49$ to 0.96$) \dagger$ & 0.65 (0.45 to 0.94$) \dagger$ \\
\hline
\end{tabular}

*Self-reported number of medicines was missing at both time points for two(0.2\%) participants who were excluded from this analysis. †z score $\mathrm{p}<0.05$.

$\ddagger$ Continuous variables with OR for each one unit increase in the number of regular medicines/chronic conditions.

or the types of ADEs and causative drug classes may also differ by age group. ${ }^{35}$

\section{Strengths and limitations}

This appears to be the first study to assess the prospective relationship between PIP in middle-aged people and adverse outcomes for patients. This large longitudinal study which included methods to account for time-varying covariates allowed for improved confounding control to reduce potential bias. A further strength is that all 22 PROMPT criteria were applied to determine exposure to PIP. Dispensing data was employed, which is likely more reliable than self-reported medicines use. ${ }^{36}$ However this required an assumption that dispensed drugs were actually taken by patients, and information on non-prescription medicines use was lacking which may have affected the reliability of criteria where over-the-counter treatment is available.

A limitation of this study was that ED and GP visits were based solely on participant recall over a 12-month period, and no validation against administrative records was possible. While there is the potential for under-reporting due to long recall time period, ${ }^{37}$ self-reported healthcare utilisation is valid across socioeconomic groups, ${ }^{38}$ and hospital ED visits are often recalled with more than $90 \%$ accuracy. ${ }^{39}$ Although the analytical approach was the same as in a previous study of older participants in TILDA, the present analysis may have been underpowered to detect an effect on healthcare use, given lower GP and ED visit rates among middle-aged people. Similarly, the effect of PIP on QoL observed in older people was modest, ${ }^{27}$ and may not have been detectable in a middle-aged cohort of this size. However, this study did have sufficient power to detect significant associations of number of regular medicines, number of conditions and level of education with healthcare utilisation. A fixed 12-month exposure period was used prior to outcome measurement, which in the case of healthcare use was also over a fixed period of 12 months, so any effect of PIP would have to be sustained beyond the exposure period in order to be detected. The outcomes investigated here may not be sensitive to the effect of PROMPT criteria as, for example, the CASP-R12 is a measure of QoL rather than health-related QoL and so may not reflect health status changes. For both ED and GP visits, the reason for encounter was not known and so, for example, GP visits involving a full assessment and visits to collect a prescription could not be distinguished.

As participants of this study had to be eligible for the GMS scheme, this introduces a bias as socioeconomically deprived individuals are more likely to have multimorbidity and PIP, ${ }^{314}$ and therefore the prevalence of PIP is likely to be an overestimate of prevalence in the general population. Although the greater level of exposure provides more power to detect an effect, a limitation is that this study could not evaluate if the relationship between PIP exposure and outcomes differs across levels of SES.

\section{Practice and policy implications}

It is possible that PIP may cause adverse effects in middleaged people but that these may not be serious enough to result in seeking healthcare or a sustained effect on QoL. Middle-aged people are likely to have superior physiological reserve and so be more capable of sustaining the adverse effects of such prescribing than older individuals. ${ }^{10}$ For example, lack of physiological changes to the gastric lining may offer natural resistance to the ulcerogenic effects of long-term NSAIDs. ${ }^{40}$ Less postural hypotension and improved balance may protect against falls due to benzodiazepines and lower prevalence of osteoporosis reduces the chance of fracture. ${ }^{42}$

Whether intervening to reduce PIP in middle-aged patients would improve patient outcomes such as healthcare use and QoL remains to be seen. Further research should be conducted to provide more evidence on whether PIP in this age group is associated with adverse outcomes. Other end points such as medication-related hospital admissions, ADEs and health-related QoL should be evaluated as ED visits, GP visits and QoL may not be 


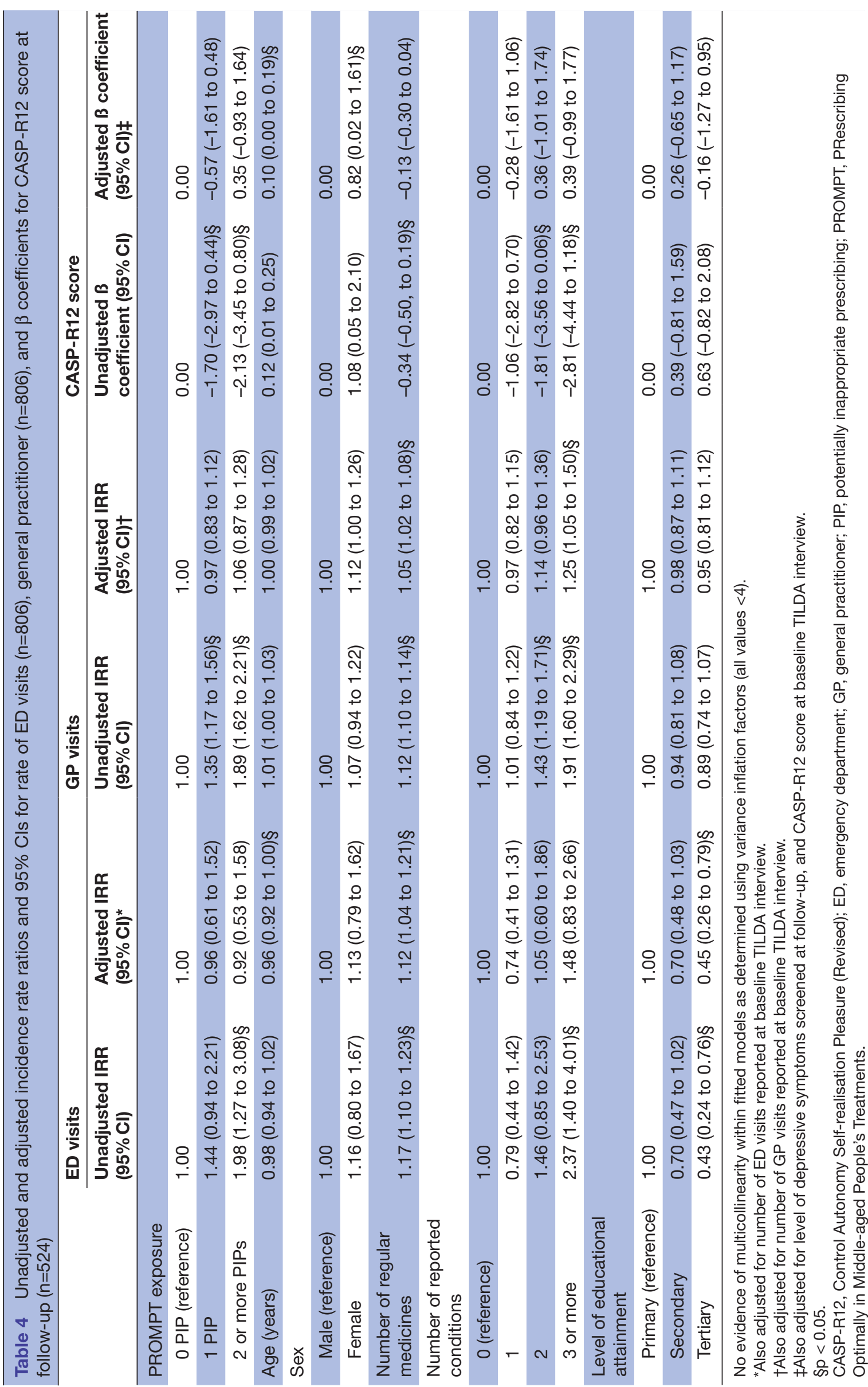


sensitive to the effects of PIP in this age group. If interventions are being introduced to optimise medicines in older patients, because PROMPT includes similar indicators to other PIP criteria lists, there may be economies of scale to also apply these interventions to the middle-aged group, if using a computer decision support system, for example. Most individual PROMPT criteria had a prevalence of less than $2 \%$ in this study, and so researchers and practitioners using this tool in the future should be cognisant of this and could consider focusing on a subset of high prevalence or high-risk criteria.

It is also important to consider that while a PIP drug may not have an adverse effect on patients in middle age, once a medicine is initiated, it can often persist into older age when the likelihood of harm may increase. ${ }^{43}$ Discontinuing an inappropriate medicine may be more feasible after a shorter period of use, as is the case with benzodiazepines due to dependence. ${ }^{44}$ Similarly, withdrawal effects can be worse after prolonged use and the impact of these may be less serious in younger patients rather than in older individuals. ${ }^{45}$

Using an arbitrary age cut-off of 65 years alone to identify those vulnerable to medication problems may not be appropriate as, although older patients may be more susceptible to the effects of PIP than middle-aged people, some younger individuals may also be at high risk. Given the increasing burden of multimorbidity, with high variation within age groups which can be explained by disparity in socioeconomic status and other factors, ${ }^{3}$ medicines optimisation efforts may be better aligned with illness burden and deprivation rather than just chronological age. ${ }^{46}$ This is reinforced by the present study which found that numbers of medicines and conditions were associated with poorer outcomes in this middle-aged cohort. Even within this relatively deprived group, those with only primary education had higher rates of ED visits compared with those with tertiary education.

\section{CONCLUSIONS}

A large proportion of middle-aged people in this cohort had PIP at baseline and this increased after 2 years of follow-up. While no evidence of a significant relationship between the PROMPT criteria and the outcomes of healthcare use and QoL was found, numbers of comorbidities and medicines were associated with these outcomes in this deprived middle-aged cohort. Further research should examine the effects of the PROMPT criteria on these and other outcomes and the burden of PIP among middle-aged people should be considered when implementing strategies to improve medicines use in other patient groups.

\section{Twitter@FrankMoriarty}

Acknowledgements We would like to thank the HSE-PCRS for providing access to the administrative pharmacy claims database used for this study and the participants of TILDA.

Contributors $\mathrm{FM}, \mathrm{CC}, \mathrm{KB}, \mathrm{CH}$ and TF contributed to the conception of this study. FM, CC, KB and TF designed this study and data were provided by KB (HSE-PCRS) and RAK (TILDA). FM carried out the statistical analysis and FM, CC, KB and TF interpreted the data. The manuscript was drafted by FM and all authors were involved in the critical revision of this and approval of the final manuscript.

Funding This work was supported by the Health Research Board of Ireland (HRB) through the HRB PhD Scholars Programme in Health Services Research (grant no. PHD/2007/16) and the HRB Centre for Primary Care Research (grant no. HRC/2007/1). KB and CC were supported by HRB (grant HRB RLA15-1579) TILDA is supported by Department of Health and Children, The Atlantic Philanthropies and Irish Life.

Competing interests All authors have completed the ICMJE Unified Competing Interest form and declare no competing intersets.

Ethics approval Faculty of Health Sciences Ethics Committee, Trinity College Dublin.

Provenance and peer review Not commissioned; externally peer reviewed. Data sharing statement № additional data available.

Open Access This is an Open Access article distributed in accordance with the Creative Commons Attribution Non Commercial (CC BY-NC 4.0) license, which permits others to distribute, remix, adapt, build upon this work non-commercially, and license their derivative works on different terms, provided the original work is properly cited and the use is non-commercial. See: http://creativecommons.org/ licenses/by-nc/4.0/

(c) Article author(s) (or their employer(s) unless otherwise stated in the text of the article) 2017. All rights reserved. No commercial use is permitted unless otherwise expressly granted.

\section{REFERENCES}

1. Gallagher P, Barry P, O'Mahony D. Inappropriate prescribing in the elderly. J Clin Pharm Ther 2007;32:113-21.

2. O'Mahony D, Gallagher PF. Inappropriate prescribing in the older population: need for new criteria. Age Ageing 2008;37:138-41.

3. Barnett K, Mercer SW, Norbury M, et al. Epidemiology of multimorbidity and implications for health care, research, and medical education: a cross-sectional study. Lancet 2012;380:37-43.

4. Guthrie B, Makubate B, Hernandez-Santiago V, et al. The rising tide of polypharmacy and drug-drug interactions: population database analysis 1995-2010. BMC Med 2015;13:74.

5. Vestal RE. Aging and pharmacology. Cancer 1997;80:1302-10.

6. Gurwitz JH, Avorn J. The ambiguous relation between aging and adverse drug reactions. Ann Intern Med 1991;114:956-66.

7. Grymonpre RE, Mitenko PA, Sitar DS, et al. Drug-associated hospital admissions in older medical patients. J Am Geriatr Soc 1988;36:1092-8.

8. Bégaud B, Martin K, Fourrier A, et al. Does age increase the risk of adverse drug reactions? Br J Clin Pharmacol 2002;54:550-2.

9. Cooper JA, Ryan C, Smith SM, et al. The development of the PROMPT (PRescribing Optimally in Middle-aged People's Treatments) criteria. BMC Health Serv Res 2014;14:484.

10. Bowie MW, Slattum PW. Pharmacodynamics in older adults: a review. Am J Geriatr Pharmacother 2007;5:263-303.

11. Bhala N, Emberson J, Merhi A, et al. Vascular and upper gastrointestinal effects of non-steroidal anti-inflammatory drugs: meta-analyses of individual participant data from randomised trials. Lancet 2013;382:769-79.

12. Spinewine A, Schmader KE, Barber N, et al. Appropriate prescribing in elderly people: how well can it be measured and optimised? Lancet 2007;370:173-84.

13. Whelan BJ, Savva GM. Design and methodology of the Irish Longitudinal Study on Ageing. J Am Geriatr Soc 2013;61:S265-S268.

14. Cooper JA, Moriarty F, Ryan C, et al. Potentially inappropriate prescribing in two populations with differing socio-economic profiles: a cross-sectional database study using the PROMPT criteria. Eur $J$ Clin Pharmacol 2016;72:583-91.

15. von Elm E, Altman DG, Egger M, et al. The Strengthening the Reporting of Observational Studies in Epidemiology (STROBE) statement: guidelines for reporting observational studies. PLoS Med 2007;4:e296.

16. Zeger SL, Liang KY, Albert PS. Models for longitudinal data: a generalized estimating equation approach. Biometrics 1988;44:1049-60.

17. Galvin R, Moriarty F, Cousins G, et al. Prevalence of potentially inappropriate prescribing and prescribing omissions in older lrish adults: findings from The Irish LongituDinal Study on Ageing study (TILDA). Eur J Clin Pharmacol 2014;70:599-606. 
18. Haider SI, Johnell K, Thorslund M, et al. Trends in polypharmacy and potential drug-drug interactions across educational groups in elderly patients in Sweden for the period 1992 - 2002. Int J Clin Pharmacol Ther 2007:45:643-53.

19. Grundy E, Holt G. The socioeconomic status of older adults: how should we measure it in studies of health inequalities? J Epidemiol Community Health 2001;55:895-904.

20. Hyde M, Wiggins RD, Higgs $P$, et al. A measure of quality of life in early old age: the theory, development and properties of a needs satisfaction model (CASP-19). Aging Ment Health 2003;7:186-94.

21. Sexton E, King-Kallimanis BL, Conroy RM, et al. Psychometric evaluation of the CASP-19 quality of life scale in an older Irish cohort. Qual Life Res 2013;22:2549-59.

22. Cahir C, Bennett K, Teljeur C, et al. Potentially inappropriate prescribing and adverse health outcomes in community dwelling older patients. Br J Clin Pharmacol 2014;77:201-10.

23. Robins JM, Hernán MA, Brumback B. Marginal structural models and causal inference in epidemiology. Epidemiology 2000;11:550-60.

24. Fewell Z, Hernán M, Wolfe F, et al. Controlling for timedependent confounding using marginal structural models. Stata J 2004:4:402-20.

25. Guthrie B, McCowan C, Davey P, et al. High risk prescribing in primary care patients particularly vulnerable to adverse drug events: cross sectional population database analysis in Scottish general practice. BMJ 2011;342:d3514.

26. Hill-Taylor B, Sketris I, Hayden J, et al. Application of the STOPP/START criteria: a systematic review of the prevalence of potentially inappropriate prescribing in older adults, and evidence of clinical, humanistic and economic impact. J Clin Pharm Ther 2013;38:360-72.

27. Moriarty F, Bennett K, Cahir C, et al. Potentially inappropriate prescribing according to STOPP and START and adverse outcomes in community-dwelling older people: a prospective cohort study. $\mathrm{Br} J$ Clin Pharmacol 2016;82:849-57. Epub ahead of print.

28. Kripke DF, Klauber MR, Wingard DL, et al. Mortality hazard associated with prescription hypnotics. Biol Psychiatry 1998;43:687-93.

29. Taché SV, Sönnichsen A, Ashcroft DM. Prevalence of adverse drug events in ambulatory care: a systematic review. Ann Pharmacother 2011;45:977-89.

30. Eguale T, Buckeridge DL, Verma A, et al. Association of off-label drug use and adverse drug events in an adult population. JAMA Intern Med 2016;176:55-63.

31. Bourgeois FT, Shannon MW, Valim C, et al. Adverse drug events in the outpatient setting: an 11-year national analysis. Pharmacoepidemiol Drug Saf 2010;19:901-10.

32. Martin RM, Biswas PN, Freemantle SN, et al. Age and sex distribution of suspected adverse drug reactions to newly marketed drugs in general practice in England: analysis of 48 cohort studies. Br J Clin Pharmacol 1998;46:505-11.

33. Bates DW. Patient risk factors for adverse drug events in hospitalized patients. Arch Intern Med 1999;159:2553-60.

34. Boeker EB, Ram K, Klopotowska JE, et al. An individual patient data meta-analysis on factors associated with adverse drug events in surgical and non-surgical inpatients. Br J Clin Pharmacol 2015;79:548-57.

35. Chen YC, Huang $\mathrm{HH}$, Fan JS, et al. Comparing characteristics of adverse drug events between older and younger adults presenting to a Taiwan emergency department. Medicine 2015;94:e547.

36. Richardson K, Kenny RA, Peklar J, et al. Agreement between patient interview data on prescription medication use and pharmacy records in those aged older than 50 years varied by therapeutic group and reporting of indicated health conditions. J Clin Epidemiol 2013;66:1308-16.

37. Bhandari A, Wagner T. Self-reported utilization of health care services: improving measurement and accuracy. Med Care Res Rev 2006;63:217-35.

38. Reijneveld SA, Stronks K. The validity of self-reported use of health care across socioeconomic strata: a comparison of survey and registration data. Int J Epidemiol 2001;30:1407-14.

39. Short ME, Goetzel RZ, Pei X, et al. How accurate are self-reports? Analysis of self-reported health care utilization and absence when compared with administrative data. J Occup Environ Med 2009;51:786-96.

40. Lovat LB. Age related changes in gut physiology and nutritional status. Gut 1996;38:306-9.

41. Newton JL. Changes in upper gastrointestinal physiology with age. Mech Ageing Dev 2004;125:867-70.

42. Todd C, Skelton D. What are the main risk factors for falls amongst older people and what are the most effective interventions to prevent these falls? WHO Regional Office for Europe, Copenhagen, Denmark 2004.

43. Anderson K, Stowasser D, Freeman C, et al. Prescriber barriers and enablers to minimising potentially inappropriate medications in adults: a systematic review and thematic synthesis. BMJ Open 2014;4:e006544.

44. Cumming RG, Le Couteur DG. Benzodiazepines and risk of hip fractures in older people. CNS Drugs 2003;17:825-37.

45. Ailabouni NJ, Nishtala PS, Mangin D, et al. General practitioners' insight into deprescribing for the multimorbid older individual: a qualitative study. Int J Clin Pract 2016;70:261-76.

46. Wallace E, Salisbury C, Guthrie B, et al. Managing patients with multimorbidity in primary care. BMJ 2015;350:h176. 